\title{
Reflets
}

Revue ontaroise d'intervention sociale et communautaire

\section{Cuisines collectives : modèle alternatif d'organisation sociale et économique}

\section{Manon Mongrain}

Volume 3, numéro 1, printemps 1997

Enfance et familles en contexte d'appauvrissement

URI : https://id.erudit.org/iderudit/026152ar

DOI : https://doi.org/10.7202/026152ar

Aller au sommaire du numéro

Éditeur(s)

Reflets : Revue ontaroise d'intervention sociale et communautaire

ISSN

1203-4576 (imprimé)

1712-8498 (numérique)

Découvrir la revue

Citer cet article

Mongrain, M. (1997). Cuisines collectives : modèle alternatif d'organisation sociale et économique. Reflets, 3(1), 80-100. https://doi.org/10.7202/026152ar

\section{Résumé de l'article}

De plus en plus récupérée dans le contexte de la sécurité alimentaire, la cuisine collective se débat contre de vives poussées vers la marginalisation, la stigmatisation et l'exclusion. Cet article présente le portrait de cuisines collectives de la Basse-ville d'Ottawa qui s'inscrivent en rupture de la vision souvent trop réductionniste de la cuisine collective. Les artisanes et les artisans de cet aménagement collectif le conçoivent comme une étape acheminant à l'autonomie plutôt que comme une fin en soi avec pour objectifs ultimes de satisfaire des besoins nutritifs et d'assurer le développement de bonnes habitudes alimentaires. Animée d'un concept central, soit le statut d'actrice et d'acteur, la cuisine collective se positionne parmi les sources d'influence qui tentent de définir sa nature pour privilégier une définition socialisée, où la reprise du pouvoir est l'objectif ultime.
Tous droits réservés (C Reflets : Revue ontaroise d'intervention sociale et communautaire, 1997
Ce document est protégé par la loi sur le droit d'auteur. L'utilisation des services d'Érudit (y compris la reproduction) est assujettie à sa politique d'utilisation que vous pouvez consulter en ligne.

https://apropos.erudit.org/fr/usagers/politique-dutilisation/ 


\section{Cuisines collectives : modèle alternatif d'organisation sociale et économique}

De plus en plus récupérée dans le contexte de la sécurité alimentaire, la cuisine collective se débat contre de vives poussées vers la marginalisation, la stigmatisation et l'exclusion. Cet article présente le portrait de cuisines collectives de la Basse-ville d'Ottawa qui s'inscrivent en rupture de la vision souvent trop réductionniste de la cuisine collective. Les artisanes et les artisans de cet aménagement collectif le conçoivent comme une étape acheminant à l'autonomie plutôt que comme une fin en soi avec pour objectifs ultimes de satisfaire des besoins nutritifs et d'assurer le développement de bonnes habitudes alimentaires. Animée d'un concept central, soit le statut d'actrice et d'acteur, la cuisine collective se positionne parmi les sources d'influence qui tentent de définir sa nature pour privilégier une définition socialisée, où la reprise du pouvoir est l'objectif ultime.

\section{Manon Mongrain \\ École de service social, Université d'Ottawa}

Entourés de l'opulence d'une ville capitale, des femmes et des hommes francophones de la Basse-ville d'Ottawa s'activent au sein des cuisines collectives afin d'échapper à la faim et à la charité, la faim qui les assaille et la charité qui les dépouille de leur dignité. Ces femmes et ces hommes sont coincés entre les structures sociales qui les infériorisent et les mesures d'assistance qui les marginalisent davantage. Partout leur statut d'actrices et d'acteurs qui confere droit et respect est nié. Devant ce non-être social, économique et politique d'individus qui vivent dans la pauvreté et de leurs entreprises souterraines, l'association coopérative constitue non pas la voie de la dérobade, mais celle de la réappropriation, voire 
"La cuisine collective doit se définir et devenir un lieu de reprise du pouvoir et de promotion de valeurs de justice sociale, sans quoi elle risque de tomber dans le piège de la marginalisation et de l'exclusion des personnes qui vivent dans la pauvreté.» de l'action. La cuisine collective doit se définir et devenir un lieu de reprise du pouvoir et de promotion de valeurs de justice sociale, sans quoi elle risque de tomber dans le piège de la marginalisation et de l'exclusion des personnes qui vivent dans la pauvreté.

Dans ce texte, nous présenterons une perception de la cuisine collective par rapport aux éléments de définition de la pauvreté et ce, à travers des concepts tels l'urgence de la faim et la sécurité alimentaire. Nous exposerons l'expérimentation d'une cuisine collective coopérative via un cadre de valeurs émergé d'une économie socialisée. L'expérience collective dont il est question s'est déployée au Centre de ressources de la Basse-ville d'Ottawa; la conception de la cuisine collective est dégagée d'un portrait dressé par l'analyse de documents et de réflexions d'intervenantes oeuvrant majoritairement à Ottawa.

\section{À la croisée de l'artère sociale-économique, de l'avenue de la coopération et de «l'entre-assistance»}

S'il est admis que l'indigence soit effectivement l'aboutissement d'une attribution inégale des ressources et du pouvoir au sein de la société, les actions visant à contrer la pauvreté doivent alors s'inscrire en rupture avec des organisations fondées sur l'injustice, la disparité et le déséquilibre (Jacques 1982: 21; Kent 1984). Attendu qu'il ne soit pas possible d'attendre patiemment la restructuration de l'ordre social et économique qui prévaut (Legault, dans Doyle et Watson 1986: 8-9), la voie de l'économie sociale offre, aux personnes marginalisées par le système, la possibilité de vivre et de participer à une organisation sociale et économique fondée sur des valeurs différentes de celles qui sont véhiculées à travers les structures sociales et économiques actuelles qui sont assujettissantes: «L'économie sociale est ressentie vivement comme une économie à taille humaine et humaniste, opposée à une économie du profit, économie pour l'économie" (Jean et Verdier, dans Boursier 1984: 36). 
La voie de l'économie sociale constitue une voie distincte de l'économie capitaliste et de l'économie planifiée. Selon François Boursier (1984:36) «le souci double de l'économie sociale reste bien de se démarquer de l'État et du libéralisme». Ses principes et valeurs la dissocient du capitalisme et son autonomie propre de l'État. S'inscrivent à l'intérieur du parapluie de l'économie sociale, toutes les organisations qui adhèrent à ces principes et qui sont instituées d'une "solidarité associative» (Boursier 1984:36).Ainsi, le modèle d'association coopérative constitue l'une des grandes lignées du secteur de l'économie sociale (Monzon Campos 1992: 52; Boursier 1984: 35). Les cuisines collectives s'inscrivent dans ce mouvement de l'économie sociale.

\section{Conceptions des cuisines collectives}

Canaux de l'affranchissement vis-à-vis de la charité alimentaire, les cuisines collectives sont reconnues comme des initiatives qui sont «d'incroyables opérations de dignité souterraines qui n'ont rien à voir avec la charité du temps des fêtes» (Émond 1992a:10).

La cuisine collective est en quelque sorte une coopérative alliant la consommation et le travail en réponse à des besoins d'ordre alimentaire, bien sûr, mais également à une variété d'autres besoins. En effet, selon Linda Couture, coordonnatrice d'un

"La cuisine collective, c'est également un laboratoire permettant d'expérimenter et de s'ouvrir à d'autres formes d'organisation sociale et économique, à d'autres valeurs. Elle est aussi un espace propice à la prise de conscience par rapport au potentiel personnel et collectif...» organisme communautaire àVal-Bélair au Québec, «... les cuisines collectives, c'est plus qu'une aide alimentaire. Elles brisent l'isolement, suscitent l'entraide et motivent les participantes dans leur désir de se prendre en main».

La cuisine collective, c'est aussi une façon de combattre l'exclusion et de maintenir sa présence sociale et économique à titre de consommatrice et de consommateur. C'est par la mise en commun des ressources et des énergies que les participantes et les participants réussissent à préserver leur pouvoir d'achat sur le marché alimentaire. De même, ces personnes échappent à l'infériorisation liée au recours à l'assistance par l'entremise des organismes de charité. La cuisine collective, c'est également un laboratoire permettant d'expérimenter et de s'ouvrir à d'autres formes d'organisation sociale et économique, à d'autres valeurs. 
Elle est aussi un espace propice à la prise de conscience par rapport au potentiel personnel et collectif (Regroupement des cuisines collectives du Québec: 1), et aux sources d'oppression extérieures. En somme, la cuisine collective constitue un précieux tremplin conduisant souvent à l'investissement et à la prise en charge personnelle et collective:

Le support et l'accroissement de l'estime personnel qui viennent avec la participation à une cuisine collective ont eu pour effet d'habiliter quelques participants à former des groupes de défense des droits des personnes à faible revenu, à militer en faveur de logement abordable (...). Quelques participants sont devenus des chefs de file de nouvelles cuisines collectives; d'autres se sont trouvé des emplois dans le domaine de l'alimentation (Kalina 1993: 13, traduction libre).

Malgré les bénéfices très appréciables de la cuisine collective, le degré d'autonomie et l'accroissement du pouvoir personnel et collectif des gens sont contenus dans la seule consommation alimentaire. C'est pourquoi il devient intéressant d'apprécier, outre les contours de la cuisine collective, le potentiel d'action susceptible de sortir de ce petit laboratoire.

La cuisine collective se matérialise au gré de la trame qui l'imagine. Les éléments de problématique ici exposés ont influencé notre vision personnelle de ce type d'aménagement collectif. Mais dans un premier temps, il nous est paru intéressant de se demander comment la participation aux cuisines collectives, qui ont une courte histoire à Ottawa-Carleton, est vécue. Quoi de plus pour ces femmes et ces hommes qui participent à une cuisine collective, que cette activité-marmiton? Dans un contexte plus large, quelles sont les forces socio-politiques qui façonnent la cuisine collective? Enfin, quels sont les enjeux qui y sont liés en terme d'objectifs et de valeurs promues?

Traitée par les professionnels et les législateurs dans le contexte de la sécurité alimentaire et des mesures à la pauvreté, cette ressource au contour vaporeux et flou est ralliée par les définisseurs 
officiels et officieux, et ce, pensons-nous, au détriment des participantes et des participants pour qui l'indéfini, l'indéterminé signifie pouvoir d'auto-définition. Les champs politique et professionnel possèdent donc une voix importante au chapitre des questions soulevées.

\section{Introduction par les voies régionales officielles de la cuisine collective à Ottawa-Carleton}

C'est en 1991, sous l'égide du ministère des Services sociaux et communautaires de l'Ontario que nait la Coalition alimentaire d'Ottawa-Carleton. Un terrain favorable à l'émergence d'un questionnement social et politique quant aux mesures gouvernementales à l'égard de la pauvreté s'aménage petit à petit. Un tout premier mandat conduit la Coalition à s'investir dans la réalisation d'un projet de recherche relatif aux solutions alternatives à la prospérité inquiétante des comptoirs alimentaires (The OttawaCarleton Coalition 1992). Néanmoins, le débat régional est véritablement soulevé au printemps 1993 sous l'impulsion du comptoir d'approvisionnement Dalhousie, mobilisé en réaction à la coupure de fonds que lui impose le Département des Services sociaux et communautaires de la Municipalité régionale d'OttawaCarleton (MROC).

L'intérêt médiatique massif et sympathique à la question de la charité des comptoirs alimentaires et l'accroissement en flèche du financement de ces mêmes programmes soulèvent un questionnement de fond au sein des départements des Services sociaux et communautaires et de la Planification de la MROC.

L'esprit des recommandations qui sont élaborées se veut une relance intéressante au niveau de la réflexion et de l'action, quoique l'enveloppe budgétaire allouée aux programmes alimentaires demeure maigre (MROC, Mémorandum 1993). En fait, l'intention derrière les recommandations vise, d'une part, à rediriger les fonds vers des résolutions à long terme plutôt que vers des solutions ponctuelles dont les mesures de charité, et d'autre part, à impliquer les diverses instances et les communautés d'une façon plus active. En d'autres mots, l'intention des départements est de lier la 
question de la faim à celle de l'indigence et d'encourager la responsabilisation sociale face à la pauvreté.

Cependant, voulant préserver les mesures de charité en place, le comité des Services sociaux ignore les recommandations les plus radicales émises par les départements chargés du dossier (MROC, Mémorandum 1993: 18), dont celle visant à rediriger les fonds vers des éléments de résolution plus en profondeur du problème de la pauvreté (emploi, action sociale, etc.). «Les banques d'alimentation peuvent respirer, la MROC va continuer à les financer» annonce France Pilon (1993: 7) dans un article publié au lendemain de la réunion du comité des Services sociaux. Certains se réjouissent et pourtant cette décision s'avère, en fait, une réitération de l'immuabilité sociale et politique face à la pauvreté.

\section{Cuisine collective : un substitut à la charité?}

"...la perspective de l'urgence alimente et perpétue le cercle vicieux de la charité, ... de telle sorte que

l'attention est complètement détournée des autres besoins engendrés par la pauvreté, et des causes sous-jacentes qui en sont responsables.»
De mal en pis, la perspective de l'urgence alimente et perpétue le cercle vicieux de la charité, cercle qui se resserre en un noeud de prostration des «bonnes intentions», de telle sorte que l'attention est complètement détournée des autres besoins engendrés par la pauvreté, et des causes sous-jacentes qui en sont responsables. La question de la faim se trouve extraite du contexte structurel au sein duquel elle s'inscrit. L'aveuglement et l'inertie sociale et politique se perpétuent.

C'est dans le cadre de cette perspective de la faim que la cuisine collective est introduite comme une alternative aux comptoirs alimentaires (Comité des Services sociaux 1993). Disons d'abord que devant la contestation de plus en plus manifeste des "pratiques samaritaines», les pratiques d'entraide deviennent pour le moins attrayantes. Considérée comme une meilleure option à la charité, la cuisine collective se voit récupérée et ralliée à l'échiquier des mesures «officielles» en réponse à la problématique de la faim.

Il y a néanmoins anguille sous roche. L'anguille, à savoir l'argument politique le plus soutenu en faveur des alternatives à la charité des comptoirs alimentaires, dénonce la dépendance qu'installe la charité chez les personnes qui vivent dans la pauvreté 
"...la cuisine collective est non seulement aliénée et dénaturée mais elle risque également de devenir un substitut à la charité, une autre procédure de dérobade à un questionnement et à des résolutions en profondeur de

l'indigence.»
(Comité des Services sociaux 1993: 8). Pour redresser cette situation, le principe de la responsabilisation des personnes face à la pauvreté qui les accable est réitéré haut et fort comme successeur à la charité en tant que précepte idéologique dans le "combat» contre la pauvreté. Appliqué à la conception des mesures, ce principe de la responsabilisation cimente les barricades sociales et politiques qui préservent le fardeau de la résolution du problème de l'indigence chez les personnes démunies. Évidemment, les pratiques d'entraide sont toutes désignées pour souscrire à ce principe de l'implication politique minimaliste. Cela étant, sous l'égide des «bonnes intentions politiques», la cuisine collective est, en fait, exploitée à des fins antagonistes aux intérêts réels et au bien-être des personnes qui vivent dans la pauvreté.

De plus, la récupération politique de la cuisine collective dans le contexte de l'extrême pauvreté et l'alliance conséquente de cette pratique avec la problématique marginalisée de la faim entraînent la stigmatisation des gens qu'elle réunit. Ainsi associée à la famine, la cuisine collective prend des airs de lieu d'exclusion, comme la soupe populaire ou le comptoir alimentaire, et se retrouve réduite à la seule dimension alimentaire, se voyant ainsi dépouillée de son caractère communautaire, de sa dignité. Bien que la cuisine collective s'avère un lieu de rencontre, elle regroupe les individus dans un espace bannissant et refermé sur lui-même. Elle devient un vase clos plutôt qu'un lieu d'inclusion sociale où sont recouvrés les statuts d'actrice et d'acteur.

Bref, sous ce couvert, la cuisine collective est non seulement aliénée et dénaturée mais elle risque également de devenir un substitut à la charité, une autre procédure de dérobade à un questionnement et à des résolutions en profondeur de l'indigence. Le fardeau du problème de la pauvreté se voit, encore une fois, refoulé et contenu dans le camp de ceux que l'on dit "pauvres". La mise à confrontation sociale et politique se soustrait d'emblée et fait place à la réitération d'un cercle fermé de la pauvreté qui met à distance les réalités d'indigence de ses sources productrices.

Devant cet état de fait, la cuisine collective devient une «alternative» de choix aux vues des décideurs politiques confrontés 
à la décadence des mesures de charité dont la moralité et l'efficacité sont de plus en plus mises en doute.

«Dans l'optique

professionnelle

s'inscrivant dans le

prolongement de la vision socio-politique, la cuisine collective est mise à profit dans le but de relancer le principe directeur de la responsabilisation individuelle vis-à-vis sa propre sécurité alimentaire.»

\section{Conception professionnelle et dominante de la cuisine collective matérialisée dans le contexte de la sécurité alimentaire}

Dans l'optique professionnelle s'inscrivant dans le prolongement de la vision socio-politique, la cuisine collective est mise à profit dans le but de relancer le principe directeur de la responsabilisation individuelle vis-à-vis sa propre sécurité alimentaire.

Ce que l'on désigne ici de "contrefaçon de la cuisine collective», soit cette même dénaturation de prescription politique et professionnelle, est étayée dans une étude historique réalisée par Dolorès Hayden (1981:176) au sujet des premières cuisines collectives en Amérique du Nord. Selon l'auteure, la mutation des cuisines communautaires du début du siècle aux États-Unis vers une définition professionnelle s'est réalisée au détriment du caractère communautaire et féministe, voire, au détriment de l'essence même de cette pratique collective.

Pendant que les professionnelles développaient l'art de l'aide mutuelle, ce qu'elles perdaient de l'idéologie socialiste, elles espéraient le retrouver dans l'efficacité; ce qu'elles perdaient de l'idéologie féministe, elles espéraient le récupérer par un statut professionnel (Hayden 1981: 177, traduction libre).

Selon ce que rapporte Hayden (1981: 152, 157), c'est ainsi que, suscitée par des avancées technologiques et des connaissances réalisées dans le domaine de l'alimentation, cette récupération professionnelle a résulté dans la métamorphose de la cuisine communautaire, devenue un laboratoire scientifique et pédagogique axé sur le savoir et le savoir-faire. Graduellement, les organisations communautaires se sont vu transformées en programmes éducatifs.

À quelques différences près, la situation actuelle des cuisines collectives est comparable à celle rapportée par Hayden. En effet, propulsée du contexte de désengagement social, économique et 
politique, l'appropriation professionnelle se vérifie à nouveau dans la réalité des cuisines collectives d'Ottawa-Carleton et d'ailleurs. Les motifs relatifs à l'avancement des connaissances ont fait place aux motifs de désengagement social, politique et économique pour faire main basse sur les initiatives provenant de la communauté.

\section{Cuisines collectives de la Basse-ville d'Ottawa}

Dans la section qui suit, nous présenterons l'analyse des résultats d'une recherche que nous avons effectuée lorsque nous avons pris part à la vie de groupe d'une cuisine collective. Notre but premier était de cerner comment les artisanes et les artisans de ces cuisines s'approprient le contenu des valeurs à travers la réalité de l'expérience collective. Nous verrons la cuisine collective devenir non seulement le forum de l'expérimentation, mais également, de la création et du renouvellement des valeurs.

La collecte de données a été réalisée par le biais d'une prise de notes descriptives et théoriques sur le terrain, tenues dans un journal de recherche et visant à dégager des pistes de compréhension (Mayer et Ouellet 1991: 420-421). Des données ont également été colligées lors d'un tour de table effectué à l'occasion d'un dîner communautaire rassemblant les artisanes et artisans des trois cuisines collectives de la Basse-ville d'Ottawa.

\section{Tabliers portés par des femmes surtout}

«Les cuisines

collectives de la Basse-

ville d'Ottawa sont

majoritairement

fréquentées par des

femmes francophones."
Les cuisines collectives de la Basse-ville d'Ottawa sont majoritairement fréquentées par des femmes francophones. D'ailleurs, à la lumière de certains auteures et auteurs qui rédigent au genre féminin en référence aux personnes qui participent aux cuisines collectives, on est à même de penser qu'il s'agit là d'une constante (Gagnon 1992: F6; Paradis 1991:6-8). Serait-ce là une vivification d'un domaine de compétence traditionnel des femmes ou bien 
plutôt l'évocation de la pauvreté des femmes? Sans conteste, c'est la pauvreté qui entrâne les femmes vers les cuisines collectives (Paradis 1991:6). Néanmoins, «...les femmes ont toujours nourri le monde, mais on n'a jamais reconnu cette compétence. Avec les cuisines collectives, on s'appuie sur leur savoir, leurs connaissances» (Paradis 1991: 8).

Susan Cox (1992: 48) observe que ce sont d'abord et surtout des femmes qui ont recours à l'assistance des comptoirs alimentaires pour se nourrir et nourrir leurs enfants. La cuisine collective étant une voie alternative à ce type d'assistance charitable, des femmes choisissent de se retrouver entre elles pour combattre ensemble et de façon différente l'indigence quotidienne à laquelle elles sont socialement et économiquement astreintes.

Pour leur part, les femmes de la Basse-ville d'Ottawa sont doublement minoritaires, une première fois à titre de femmes et une seconde fois à titre de francophones. L'étude de Maurice

"...la cuisine collective est un lieu propice à la prise de pouvoir et à la valorisation, à la seule condition qu'on $y$ reconnaisse les habiletés et le potentiel des femmes à titre de cuisinières, de gestionnaires, de planificatrices, de négociatrices, etc.»
Saint-Germain et Marc Lavoie démontre sans équivoque la situation de double oppression sociale et économique des femmes francophones hors Québec (1992:31-57). À l'issue de leurs recherches, les deux auteurs affirment que les femmes francophones en milieu minoritaire touchent des revenus moindres que ceux des hommes et moindres que ceux des femmes anglophones.

Par cette accumulation de caractéristiques faisant l'objet d'oppression, quoi de moins étonnant que de retrouver ces femmes de la Basse-ville à s'entraider au sein de pareilles initiatives? Fort heureusement, la cuisine collective est un lieu propice à la prise de pouvoir et à la valorisation, à la seule condition qu'on y reconnaisse les habiletés et le potentiel des femmes à titre de cuisinières, de gestionnaires, de planificatrices, de négociatrices, etc.

\section{Vue alternative: rapports sociaux plus équitables}

Tenter de s'échapper du cadre étroit de la sécurité alimentaire, de se démarquer des cuisines collectives où l'on considère la pauvreté économique exclusivement, voilà l'expérience des cuisines collectives de la Basse-ville d'Ottawa. En fait,l'expérience se veut 
«En fait, l'expérience se veut une alternative immédiate, un lieu informel de prise de confiance, de pouvoir et d'expérimentation de rapports sociaux plus équitables, plus justes.» une alternative immédiate, un lieu informel de prise de confiance, de pouvoir et d'expérimentation de rapports sociaux plus équitables, plus justes.

Essentiellement, ce prototype alliera trois propriétés sous le couvert de valeurs visant la reprise des statuts d'actrice et d'acteur. Il s'agit de la coopération, de la prise et du partage du pouvoir et enfin, de l'inclusion. À partir de ces fondements, la cuisine collective deviendra le produit d'une écriture collective en harmonie avec la philosophie relative à la prise de pouvoir et à l'inclusion des individus à tous les niveaux de l'expérience, incluant la définition.

\section{Entre la poire et le fromage : ambiance au sein de la cuisine}

Le partage d'une activité et la poursuite d'un but commun tangible dans le contexte informel et familier de la cuisine ont servi de cadre à l'épanouissement quasi immédiat d'un climat d'aisance et de camaraderie au sein des deux collectifs qui ont évolué au Centre de ressources de la Basse-ville d'Ottawa. L'écart entre le climat de l'activité planification versus l'activité cuisine s'est avéré particulièrement remarquable.

En effet, lors de la toute première rencontre de chacun des groupes réunis à l'activité de planification, les interactions se sont limitées à des échanges polis, orientés presque exclusivement vers les deux stagiaires en service social. Par ailleurs, il est vrai que la réunion-planification regroupe les collectifs dans un but logistique (où l'entendement commun s'établit, l'organisation s'élabore, etc...), qui peut sembler a priori rébarbatif. Aussi, bien que la familiarité se soit tranquillement installée et les interactions diversifiées entre les membres lors de l'activité de planification, l'ambiance spontanée à la camaraderie et à la confidence a prévalu aux moments de l'activité cuisine tout particulièrement. D'ailleurs, dès la toute première rencontre cuisine, on pouvait déjà entendre des rires et des plaisanteries, observer les échanges devenir confidences et ouverture de soi et ce, au sein de chacun des deux collectifs à chacune des rencontres cuisine. 
Il est vrai que les occasions d'échange sont nombreuses dans le flot d'activités nécessaires à la préparation des repas. Aussitôt les mains à la pâte, les artisanes s'interpellent d'une façon amicale et familière par leurs prénoms. Et, dépassant de loin l'ordre des échanges fonctionnels requis, une femme raconte la dernière blague apprise, une autre parle d'elle-même. De toute part, il y a échange d'anecdotes, de blagues, de confidences. Dès lors de la première activité cuisine, on penserait que tous ces individus se connaissent depuis belle lurette.

\section{Mi-figue, mi-raisin : entre la production autonome de valeurs et l'expérimentation de valeurs hétéronomes}

Il va de soi qu'enchâsser la cuisine collective dans la voie de l'économie sociale, c'est admettre l'influence d'un cadre de valeurs et un entendement initial qui, comme on l'a vu, défie et réfute à plusieurs égards le cadre de références néo-libérales. Bien qu'en réaction aux valeurs dominantes subjuguantes, la trame de l'économie socialisée et de la coopération enjoint également ses propres valeurs, ses propres convictions, ses sources d'influence. Ce que Jean-Pierre Dupuy qualifierait de valeurs hétéronomes (1980: 56). Par valeurs hétéronomes nous, référons ici aux valeurs de coopération, de reprise, de partage du pouvoir et d'inclusion.

C'est pourquoi, en continuité avec l'approche féministe, il est primordial de favoriser la prise de pouvoir, et ce, à tous les niveaux. Précisément, la production des valeurs et des idées constitue une affirmation, donc une prise de pouvoir fonda-

"...une cuisine

collective habilitante se doit d'être un lieu de production de valeurs et non exclusivement un lieu de prescription de valeurs d'usage.» mentale. En d'autres mots, une cuisine collective habilitante se doit d'être un lieu de production de valeurs et non exclusivement un lieu de prescription de valeurs d'usage. Et en somme, dans sa forme coopérative, la cuisine collective propose un cadre, des convictions, des façons de faire, mais de même, elle favorise l'appropriation des individus sur la ressource par la création d'un espace de définition et de production autonome des valeurs et des idées. 
Un cadre coopératif expérimental "pour agir de conserve»"

Bien que la coopération s'exprime ici au niveau économique par la mise en commun des ressources de chacune et de chacun et par la répartition équitable des fruits des efforts collectifs, il est important de souligner que l'esprit coopératif s'est exprimé de façon particulière au niveau du fonctionnement tout au cours de l'expérience. C'est en ce sens, notamment, que les artisanes ont été appelées à "agir de concert», soit sur le plan concret de l'opération de la cuisine collective. Or, la coopération n'est pas nécessairement intrinsèque à cet aménagement collectif qui peut inspirer le travail et les efforts parallèles autant que la transposition de valeurs coopératives dans sa logistique et son fonctionnement concret. En ce qui concerne l'expérience qui nous intéresse, chacune des étapes (planification des menus, achats, cuisson, évaluation et gestion) ont été réalisées collectivement et de façon coopérative.

"Pouvoir d'achat, pouvoir s'inclure, pouvoir s'exprimer, pouvoir socialiser, pouvoir expérimenter, pouvoir s'organiser, pouvoir sur son quotidien, pouvoir de définition, pouvoir inventer, pouvoir sur sa conscience... avoir plein pouvoir...»

\section{Nourrir le pouvoir}

Pouvoir d'achat, pouvoir s'inclure, pouvoir s'exprimer, pouvoir socialiser, pouvoir expérimenter, pouvoir s'organiser, pouvoir sur son quotidien, pouvoir de définition, pouvoir inventer, pouvoir sur sa conscience... avoir plein pouvoir...

De toute évidence, la prise et la reprise du pouvoir représentent un, sinon «le» créneau menant à la réappropriation des statuts d'actrice et d'acteur. Le partage du pouvoir conduit à l'élaboration de structures fondées sur des valeurs sociales et de justice qui sont nécessaires à la reconnaissance du potentiel de chacune et de chacun et à la sauvegarde des dignités humaines.

La coopération et le pouvoir sont intimement liés ici, et ce, tant au niveau de la prise que du partage du pouvoir. En effet, le coopératisme enjoint les conditions foncières à la prise et au partage du pouvoir. En outre, le coopératisme établit sans conteste l'appartenance du pouvoir chez toutes celles et tous ceux qui vivent, définissent, inventent et oeuvrent dans l'organisation. Par ailleurs, la coopération est fondée sur des rapports sociaux et économiques réciproques, tant et si bien que la philosophie du 
partage du pouvoir s'y trouve implantée tout naturellement, pour ne pas dire fondamentalement. De même, la concentration du pouvoir qui découle de la concentration des savoirs et des fonctions dans une organisation est endiguée par le décloisonnement de telles chasses gardées dans une organisation coopérative.

Par l'entremise du processus et de la logistique, les artisanes et les artisans auront donc l'occasion d'agir effectivement sur la ressource.Aussi, la mise en lumière du potentiel de chacune et de chacun, de même que la réduction de la distance entre les tâches dites de la sphère publique et les habiletés des artisans, renforceront l'expérience individuelle et collective d'appropriation du pouvoir.

"L'entraide économique, le développement de solidarité et les initiatives communautaires sont des stratégies efficaces pour contrer l'exclusion.»

\section{Ouverture de l'intérieur, ouverture sur l'extérieur}

Les organisations communautaires, les institutions de type coopératif peuvent jouer un rôle intégrateur puisqu'elles portent en elles le germe de la solidarité (Laflamme et al.: 1982: 270).

L'entraide économique, le développement de solidarité et les initiatives communautaires sont des stratégies efficaces pour contrer l'exclusion. Il est de reconnaissance générale, en effet, que de telles pratiques constituent, bien souvent, l'unique créneau d'accès à la vie sociale et économique pour les populations désavantagées (Giroux et Malo 1992:17). Ouverture de l'intérieur, ouverture sur l'extérieur expriment donc la double prérogative de la cuisine collective, soit d'une part, l'inclusion, l'accessibilité, la cohésion, l'appartenance et d'autre part, l'inclusion au-delà de cette même bulle.

À peu de choses près, la cuisine collective est le produit de la mise en commun d'une activité de la sphère privée, sinon isolante. C'est l'occasion pour plusieurs artisanes de sortir de la maison et de socialiser, de rencontrer de nouvelles personnes, de développer de nouveaux contacts, et ce, sans que les limites financières habituelles ne s'interposent. Bref, la cuisine collective est une voie d'intégration à l'environnement social. De fait, cet aménagement collectif est une porte sur la communauté dans laquelle elle prend place: que ce soit à travers les liens qu'elle entretient avec ses homologues, les informations qui y sont véhiculées ou encore, 
via l'implication de certaines artisanes à l'extérieur de la cuisine collective, faisant ainsi le pont entre les organisations.

Une cuisine, un lieu de rencontre où l'on tient table ouverte

"...la cuisine collective est nourrissante à plusieurs égards. Bien au-delà des besoins physiologiques, elle tente de restaurer les liens sociaux que les valeurs capitalistes, individualisantes, la privatisation et le déracinement urbain continuent d'éroder. »
Comme l'a déjà exprimé une intervenante, la cuisine collective est nourrissante à plusieurs égards. Bien au-delà des besoins physiologiques, elle tente de restaurer les liens sociaux que les valeurs capitalistes, individualisantes, la privatisation et le déracinement urbain continuent d'éroder. De plusieurs façons, la cuisine collective fait preuve d'un potentiel inclusif que l'on observe dans l'ouverture, l'appartenance, l'inclusion des réalités des artisanes et la cohésion interne.

Avant tout, la cuisine collective doit demeurer perméable pour pouvoir subsister dans un contexte en mouvance qui met en scène des situations précaires où le quotidien est fondé sur des conditions de vie et des réalités provisoires. D'ailleurs, ce sont là des éléments de compréhension déterminants de la dissolution de l'un des collectifs. La maladie, la violence faite aux femmes et les déménagements répétés sont, en effet, aux premiers rangs des motifs de sa dissolution. Néanmoins, nous avons observé la souplesse des frontières sociales du collectif toujours fonctionnel qui s'est chargé d'accueillir une artisane du groupe dissolu. Selon notre expérience, les propriétés inclusives de la cuisine collective, à savoir l'ouverture, l'appartenance et la cohésion ne sont pas un frein à l'établissement d'une solidarité interne. Au contraire, l'inclusion d'un membre nouveau est une occasion de se présenter et donc de se définir en tant que groupe et en tant qu'individu dans le groupe. C'est ainsi que chacun s'est présenté comme individu, et avec la complicité des consoeurs et des confrères, comme individu dans le groupe, créant ainsi une image collective. Des commentaires tels «lui, c'est le spécialiste du pain de viande», laissaient transparaître la complicité déjà établie. Bref, l'ouverture a fort probablement favorisé la cohésion interne et l'appartenance du fait qu'elle a placé le collectif devant son identité collective.

Enfin, un dernier volet de l'ouverture renvoie à l'inclusion des réalités des artisanes à l'intérieur de l'aménagement collectif. 
Comme lieu informel et ouvert, la cuisine s'harmonise bien aux conditions de vie qui, loin de présenter des obstacles, la définissent en réponse aux préoccupations des gens qui la vivent. Par exemple, afin de correspondre à la disponibilité des fonds et à la période de disette, la production culinaire s'inscrit au début du mois en vue de garnir les ventres à la fin du mois. Par ailleurs, les enfants des artisanes viennent de temps en temps égayer les séances de production.

"...la cuisine collective n'est pas une fin en soi. Elle est plutôt vue comme un canal qui ouvre des voies dans l'environnement social, économique et politique.»
Du coeur de la cuisine au coeur de la communauté

Suivant la vision du Centre de ressources de la Basse-ville d'Ottawa, la cuisine collective n'est pas une fin en soi. Elle est plutôt vue comme un canal qui ouvre des voies dans l'environnement social, économique et politique. Ainsi, à la base, la conception même de la cuisine collective est conduite en dehors de l'engrenage de l'exclusion collective qui n'offre, ni plus ni moins, qu'un autre espace en marge.

Telle que nous la concevons, la cuisine collective est propice à l'inclusion du fait qu'elle agit sur deux fronts. D'une part, elle est un lieu d'habilitation, de reprise de confiance, d'expérimentation de compétences sociales et de démystification des activités de la sphère publique. D'autre part, elle s'ouvre sur l'extérieur, et ce, de deux façons. D'abord, en tentant d'émerger de ses propres murs et ensuite, en favorisant l'inclusion aux activités mêmes de la communauté.

D’abord, animé par ses principes idéologiques et inspiré des cuisines collectives péruviennes ${ }^{2}$, le Centre de ressources de la Basse-ville a voulu tâter le terrain de l'expansion de la cuisine collective dans une optique économique. Plusieurs idées ont d'ailleurs circulé, allant du jardin communautaire au système d'économie alternative. En outre, un enjeu captivant dans la Basseville concerne la construction d'une maison communautaire qui se voudrait un lieu de rencontre et d'activités rapproché des citoyens et des citoyennes. Dans les cuisines collectives, le sujet est d'ailleurs particulièrement bien alimenté puisque deux artisanes sont activement impliquées dans le comité de citoyens et de 
citoyennes occupé à promouvoir et à défendre le projet. Le rayonnement des cuisines collectives et des initiatives qui y sont issues ne cessera de réchauffer le climat de la Basse-ville. Au printemps 1996, deux cuisines collectives sont toujours fonctionnelles: une à caractère culturel composée de femmes somaliennes et une composée de femmes francophones, cette dernière étant tout à fait autonome (sans intervenante). Dans la même veine, les cuisines collectives cherchent l'inclusion dans la communauté. En ce qui concerne les femmes somaliennes, leur sens de l'appartenance se fait maintenant ressentir au niveau du Centre où elles ont tranquillement pris leur aise. Pour ce qui est de la cuisine collective des femmes francophones, «la cuisine franco", elle gagne du terrain dans la communauté à travers diverses initiatives ouvertes sur l'extérieur, dont un jardin communautaire, des groupes de discussions, un comité visant à accroître la sécurité dans le quartier, un comité pro-actif de solutions contre les coupures imposées par le gouvernement Harris. De même, elles tentent d'améliorer leur qualité de vie à travers d'autres activités nées de la cogitation du dernier comité, à savoir des levées de fonds, un service de traiteur et la planification financière et logistique des vacances de la prochaine année en vue d'assurer l'acquis de ce droit.

Bref, la cuisine collective confirme ainsi ses dispositions favorables face à l'établissement et à l'entretien des liens entre les artisanes et les artisans et la communauté, d'une part, et sa faculté à établir des ponts vers les opportunités d'inclusion et de prise de pouvoir, d'autre part.

\section{Conclusion}

Pour contrer la pauvreté, nous avons malheureusement choisi de donner du poisson et d'apprendre à pêcher à des gens non reconnus à titre de pêcheur et tenus à distance du lac. De fait, en s'éloignant de l'idéal de bien-être pour régresser vers la sécurité alimentaire et ce, sous le prétexte de la dépendance et sous le 
"C'est en réaction à

l'étroitesse des

définitions de la pauvreté qui prévalent actuellement que la cuisine collective... a tenté de se définir et même de s'inscrire en rupture avec des valeurs sociales, économiques, culturelles dominantes et des rapports sociaux de sexe infériorisants qui sont largement véhiculés.» couvert de la notion de responsabilité, certaines cuisines collectives isolent, stigmatisent. C'est pourquoi l'association coopérative est une solution de choix puisqu'elle fournit les outils et la reconnaissance nécessaires à la prise en charge personnelle et collective chez la population moins nantie.Vivre et promouvoir un projet social, voilà donc ce que font les artisanes et les artisans actifs au sein des cuisines collectives habilitantes. Ces individus ont en effet repéré un moyen précieux de faire valoir leurs intérêts et leurs idées.

C'est en réaction à l'étroitesse des définitions de la pauvreté qui prévalent actuellement que la cuisine collective à laquelle nous avons eu la chance de participer a tenté de se définir et même de s'inscrire en rupture avec des valeurs sociales, économiques, culturelles dominantes et des rapports sociaux de sexe infériorisants qui sont largement véhiculés.

De cette expérience ressortent plusieurs considérations pour la pratique auprès des artisanes et des artisans réunis dans une cuisine collective. D'abord, les fondements idéologiques de la cuisine collective doivent être compris dans une perspective globale de la pauvreté. En d'autres mots, il importe d'aller aux sources et d'identifier les définitions de la pauvreté que la ressource préconise et ses valeurs sous-jacentes. Nous pouvons perpétuer l'infériorisation des individus qui vivent dans la pauvreté ou nous pouvons mettre des outils à leur disposition en vue de créer un lieu d'appartenance, un lieu de reprise des droits et statuts d'actrice et d'acteur. Dans un souci de changement social, la cuisine collective doit donc offrir un cadre de valeurs habilitantes et de rapports réciproques.

La formule coopérative présente une avenue intéressante dans la mesure où elle protège la dignité de la ressource, des artisanes et des artisans et où elle campe clairement le pouvoir chez ces derniers. La cuisine collective doit être le produit d'une écriture justement collective afin de promouvoir les paroles, les intérêts, les stratégies et les réussites des gens qui non seulement la composent mais qui la définissent également. Les artisanes et les artisans doivent être impliqués dans la définition de la cuisine 
collective à travers l'aménagement, la gestion et la production autonome devant être encouragée via l'autogestion.

En somme, la cuisine collective se doit d'éclater le cadre de la sécurité alimentaire sans quoi elle deviendra un lieu de stigmatisation, un lieu d'exclusion. Elle doit se resocialiser si elle veut travailler à restaurer les droits et statuts d'actrice et d'acteur, reconstruire les tissus sociaux des communautés et agir à titre d'agent d'inclusion et de changement plutôt qu'à titre d'agent d'adaptation.

\section{Bibliographie}

AUTES, Michel (1985). «Les mots et les pauvres», dans La pauvreté: une approche plurielle, sous la dir. de A. FRACASSI; M.F. MARQUES et J.WALTER. Paris, Les Éditions ESF, 219-232.

BOURSIER, François (1984). L'économie sociale: mythes et réalités, Lyon, Chronique sociale.

CASTEL, Robert (1995). «L'avènement d'un individualisme négatif», Magazine littéraire, vol. 334, $16-22$.

CHARTIER, Roger (1985). «La pauvreté à l'âge moderne: définitions, représentations, institutions», dans A. Fracassi, M.F. Marques et J.Walter, éd. La pauvreté: une approche plurielle, Paris, Les Éditions ESF, 25-42.

COMITÉ DES SERVICES SOCIAUX (1993). Ordre du jour, 17 juin 1993, Ottawa, Municipalité régionale d'Ottawa-Carleton, doc. minéo.

COMITÉ DES SERVICES SOCIAUX (1993). Mémorandum, Ottawa, Municipalité régionale d'Ottawa-Carleton, doc. minéo.

COMITÉ D'EXAMEN DE L'AIDE SOCIALE (1988). Transitions, Toronto, Publications Ontario.

CONSEIL DE PLANIFICATION SOCIALE D'OTTAWA-CARLETON (1987) Analyse des services d'urgence fournissant nourriture, vêtements et hébergement à Ottawa-Carleton, Ottawa, Conseil de Planification sociale d'Ottawa-Carleton.

DÉPARTEMENT DE SANTÉ (1992). Collective Kichens Pilot Program, Program Evaluation Summary, Municipalité régionale d'Ottawa-Carleton, doc. minéo.

DOYLE, Robert etValérie Watson (1986). Rapport de la conférence nationale sur la faim, Toronto, Social Panning Council of Metropolitan Toronto.

DUPUY, Jean-Pierre (1980). «Bien-être et autonomie», Futuribles, vol. 32, 53-58.

ÉMOND, Ariane (1992). «La faim des autres», Le Devoir, mercredi le 16 décembre 1992, A10.

FORTIN, Denis (1988). Riches contre pauvres deux poids deux mesures ou de l'aide sociale aux plus démunis à l'assistance cachée pour les bien-nantis: au passage de l'État-providence à l'État-provigo!, Québec, Les Éditions Autogestionnaires.

GAGNON, Damien (1992). «Des grands-mères s'impliquent dans une cuisine collective», Le Soleil, samedi le 11 juillet 1992, f6. 
GAZIER, Bernard (1985). «Thèse explicative : la pauvreté résiduelle, bref examen des conceptions libérales», dans A. Fracassi, M.F. Marques et J. Walter, éd. La pauvreté: une approche plurielle, Paris, Les Éditions ESF, 67-74.

GIROUX, Nicole et Marie-Claire MALO (1992). «Une organisation différente: la coopérative», Femmes d'action, vol. 22, no. 1, 16-17.

HAYDEN, Dolorès (1981). The Grand Domestic Revolution, Massachussetts, The Massachussetts Institute of Technology.

HIERNAUX, Jean-Pierre (1985). "Thèse explicative: pauvreté, paupérisation et dimensions structurelles», dans A. Fracassi, M.F. Marques et J. Walter, éd. La pauvreté: une approche plurielle, Paris, Les Éditions ESF, 75-83.

JACK, Carolyn (1991). «Food Banks and the Politics of Hunger», The Canadian Forum, vol. 40, no. 208, 5-11.

JACQUES, François (1982). «Coopération et valeurs de civilisation», dans Marcel Laflamme, éd. Le projet coopératif québécois, Chicoutimi, Gaëtan Morin éditeur, 1-34.

JOIN-LAMBERT, Louis (1985). «Thèse explicative : la pauvreté persistante», dans dans A. Fracassi, M.F. Marques et J. Walter, éd. La pauvreté : une approche plurielle, Paris, Les Éditions ESF, 56-66.

KAMLOOPS FOODSHARE (1993). Community Kitchens, A Health Promotion Program to Improve the Food Security of Low-income Families, Final Evaluation Report, Kamloops, Health Research Foundation.

KENT, George (1984). The Political Economy of Hunger, New York, Preager Publishers.

LAFLAMME, Marcel et al. (1982). Le projet coopératif québécois : un projet social, Chicoutimi, gaëtan morin éditeur.

LAFLEUR, Bruno (1991). Dictionnaire des locutions idiomatiques françaises, Montréal, Éditions du Renouveau pédagogique Inc.

LAFRENIÈRE, Gérard (1987). La coopérative et les Franco-Ontariens, Sherbrooke, Université de Sherbrooke, Institut de recherche et d'enseignement pour les coopératives.

LAVOIE, Marc et Maurice SAINT-GERMAIN (1990). Statut économique relatif des francophones au Québec en 1985-1986, Ottawa, Département des sciences économiques.

LIPSKY, Micheal et Steven Rathgeb SMITH (1989). «When Social Problems are Treated as Emergencies", Social Service Review, vol. 63, 1.

MAYER, Robert et Francine OUELLET (1991). Méthodologie de recherche pour les intervenants sociaux, Boucherville, Gaëtan Morin éditeur.

MONZON CAMPOS, José Luis (1992). «L'économie sociale : troisième secteur d'un système en mutation», dans Jacques Defourny et José Luis Monzon Campos, éd. Économie sociale: entre économie capitaliste et économie publique, 13-19.

Municipalité régionale d'Ottawa-Carleton (1988). A demographic profile by neighbourhood, Ottawa, doc. mineo.

NELSON, Janet (1978). «Peri-urban malnutrition, a neglected problem, patterns and approaches», Les carnets de l'enfance, 43, 25-46.

NORMAN, Diane (1990). Collective Kitchens, How to set them up and make them work, Montéal, Regroupement des Cuisines Collectives du Québec.

PARADIS, France (1991). "Cuisines collectives : bien plus que la simple popote», La gazette des femmes, vol. 13, no 3, 6-8. 
PILON, France (1993). "La MROC continuera de contribuer au financement des banques alimentaires», Le Droit, samedi le 13 juin 1993, 7.

PLANNING COMMITTEE OFTHE COLLECTIVE KITCHEN COMMUNITY MEETING (1993). Collective Kitchens Community Meeting, Ottawa, doc. minéo.

REDJEB, Belhassen (1990). Évolution des formes d'assistance au Québec, Laval, Éditions Beauchemin.

REGROUPEMENT DES CUISINES COLLECTIVES DU QUÉBEC. Guide du fonctionnement des cuisines collectives, Montréal, doc. mineo.

RICHES, Graham (1986). «Les banques d'aliments et l'effondrement des garanties minimales de l'assistance sociale au Canada", Revue internationale d'action communautaire, vol. 16, 161-170.

THE OTTAWA-CARLETON FOOD COALITION PROJECT (1992). Overdrawn at the Food Banks: The Hunger Crisis in Ottawa-Carleton, Ottawa, Gloucester Centre for Community Resources.

VAILLANCOURT, Yves (1990). "À Ottawa: une stratégie interventionniste de construction de l'État-providence», dans L'évolution des politiques sociales au Québec, 1940-1960, Montréal, Les Presses de l'Université de Montréal.

WEBBER, Marlene (1992). Food for Thought, Toronto, Coach House Press Toronto.

\section{Notes}

1. «Ensemble; de compagnie; avec entente. D'origine maritime : se disait de deux bateaux qui se suivaient afin de pouvoir se porter secours l'un l'autre». (Lafleur 1991: 144).

2. Les cuisines collectives péruviennes prennent place dans un environnement social et économique beaucoup plus large que celui que nous connaissons ici. Par exemple, la cuisine collective peut être impliquée dans l'approvisionnement de médicaments et la production artisanale commerciale (Conférence d'artisanes péruviennes au Centre de santé du Centre-ville d'Ottawa). 